

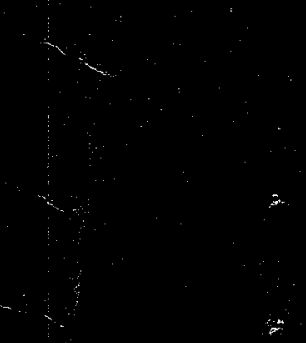


LBL -37842

UC 413

\title{
Reducible Emission Probabilities and Thermal Scaling in Multifragmentation
}

\author{
L.G. Moretto, L. Phair, K. Tso, R. Ghetti, N. Colonna, W. Skulski, \\ G.J. Wozniak
}

Nuclear Science Division, Lawrence Berkeley National Laboratory University of California, Berkeley, CA 94720, USA

D.R. Bowman, N. Carlin, M. Chartier, R.T. de Souza, C.K. Gelbke, W.G. Gong, W.C. Hsi, Y.D. Kim, M.A. Lisa, W.G. Lynch, G.F. Peaslee, C. Schwarz, M.B. Tsang, F. Zhu

National Superconducting Cyclotron Laboratory and Department of Physics and Astronomy, Michigan State University, East Lansing, MI 48824-132I, USA

August 1995

This work was supported by the Director, Office of Energy Research Division of Nuclear Physics of the Office of High Energy and Nuclear Physics of the U.S. Department of Energy under Contract DE-AC03-76SF00098 
(2) 


\title{
Reducible Emission Probabilities and Thermal Scaling in Multifragmentation
}

\author{
L.G. Moretto, L. Phair, K. Tso, R. Ghetti, N. Colonna ${ }^{a}$, W. Skulski ${ }^{b}$, G.J. Wozniak \\ Nuclear Science Division \\ Lawrence Berkeley Laboratory, Berkeley, CA 94720
}

D.R. Bowman ${ }^{c}$, N. Carlin ${ }^{d}$, M. Chartiere, R.T. de Souza ${ }^{f}$, C.K. Gelbke, W.G. Gong ${ }^{g}$, W.C. $\mathrm{Hsi}^{f}$, Y.D. $\mathrm{Kim}^{h}$, M.A. Lisa ${ }^{g}$, W.G. Lynch, G.F. Peaslee, C. Schwarz ${ }^{j}$, M.B. Tsang, F. Zhu ${ }^{k}$

National Superconducting Cyclotron Laboratory

and Department of Physics and Astronomy

Michigan State University, East Lansing, MI 48824-1321, USA

\begin{abstract}
Intermediate-mass-fragment multiplicity distributions for a variety of reactions at intermediate energies are shown to be binomial and thus reducible at all measured transverse energies. From these distributions a single binary event probability can be extracted that has a thermal dependence. A strong thermal signature is also found in the charge distributions. The $n$-fold charge distributions are reducible to the 1 fold charge distributions through a simple scaling that is dictated by fold number and charge conservation.
\end{abstract}

\section{Introduction}

At low excitation energies, complex fragments are emitted with low probability by a compound nucleus mechanism [1,2]. At increasingly larger energies, the probability of complex fragment emission increases dramatically, until several fragments are observed within a single event [3-5]. The nature of this multifragmentation process is at the center of much current attention. For example, the time-scale of fragment emission and the associated issue of sequentiality versus simultaneity are the objects of intense theoretical [3-8] and experimental [9-17] study.

Recent experimental work $[18,19]$ has shown that the excitation functions for the production of two, three, four, etc. fragments give a characteristically linear Arrhenius plot, suggesting a statistical energy dependence. 


\section{Reducibility}

A fundamental issue, connected in part to those mentioned above, is that of reducibility: can multifragmentation be reduced to a combination of (nearly) independent emissions of fragments? More to the point, can the probability for the emission of $n$ fragments be reduced to the emission probability of just one fragment?

Recently, it has been experimentally observed in many reactions that for any value of the transverse energy $E_{t}$, the $n$-fragment emission probability $P_{n}$ is reducible to the one-fragment emission probability $p$ through a binomial distribution [20]

$$
P_{n}^{m}=\frac{m !}{n !(m-n) !} p^{n}(1-p)^{m-n} .
$$

This empirical evidence indicates that multifragmentation can be thought of as a special combination of nearly independent fragment emissions. The binomial combination of the elementary probabilities points to a combinatorial structure associated with a time-like or space-like one-dimensional sequence. It was also found that the $\log$ of such one-fragment emission probabilities $(\log p)$ plotted vs $1 / \sqrt{E_{t}}$ (Arrhenius plot) gives a remarkably straight line. This linear dependence is strongly suggestive of a thermal nature for $p$,

$$
p=e^{-B / T}
$$

under the assumption that the temperature $T \propto \sqrt{E^{*}}$ where $E^{*}$ is the excitation energy. Examples of the binomial decomposition of the $n$-fragment emission probabilities $P_{n}$ into a one-fragment emission probability $p$, and the resulting Arrhenius plot for $p$ is given in Fig. 1. The extraordinary quantitative agreement between the calculations and the experimental data confirms the binomiality of the multifragmentation process.

The more directly interpretable physical parameter contained in this analysis is the binary barrier $B$ (proportional to the slope of the Arrhenius plot in Fig. 1). One may wonder why a single binary barrier suffices, since mass asymmetries with many different barriers may be present. Let us consider a barrier distribution as a function of mass asymmetry $x$ of the form $B=B_{0}+a x^{n}$, where $B_{0}$ is the lowest barrier in the range considered. Then,

$$
p=\frac{\Gamma}{\hbar \omega_{0}}=\int e^{-B_{0} / T} e^{-a x^{n} / T} d x \cong\left(\frac{T}{a}\right)^{1 / n} e^{-B_{0} / T}
$$

Thus the simple form of Eq. (2) is retained with a small and renormalizable preexponential modification.

One possible interpretation of the reducibility discussed above is sequential decay with constant probability $p$. Assuming that the (small) fragments, once produced do not generate additional fragments or disappear, the binomial distribution follows directly. In this framework, it is possible to translate the probability $p$ into the mean 
$\mathrm{E} / \mathrm{A}=50 \mathrm{MeV}$

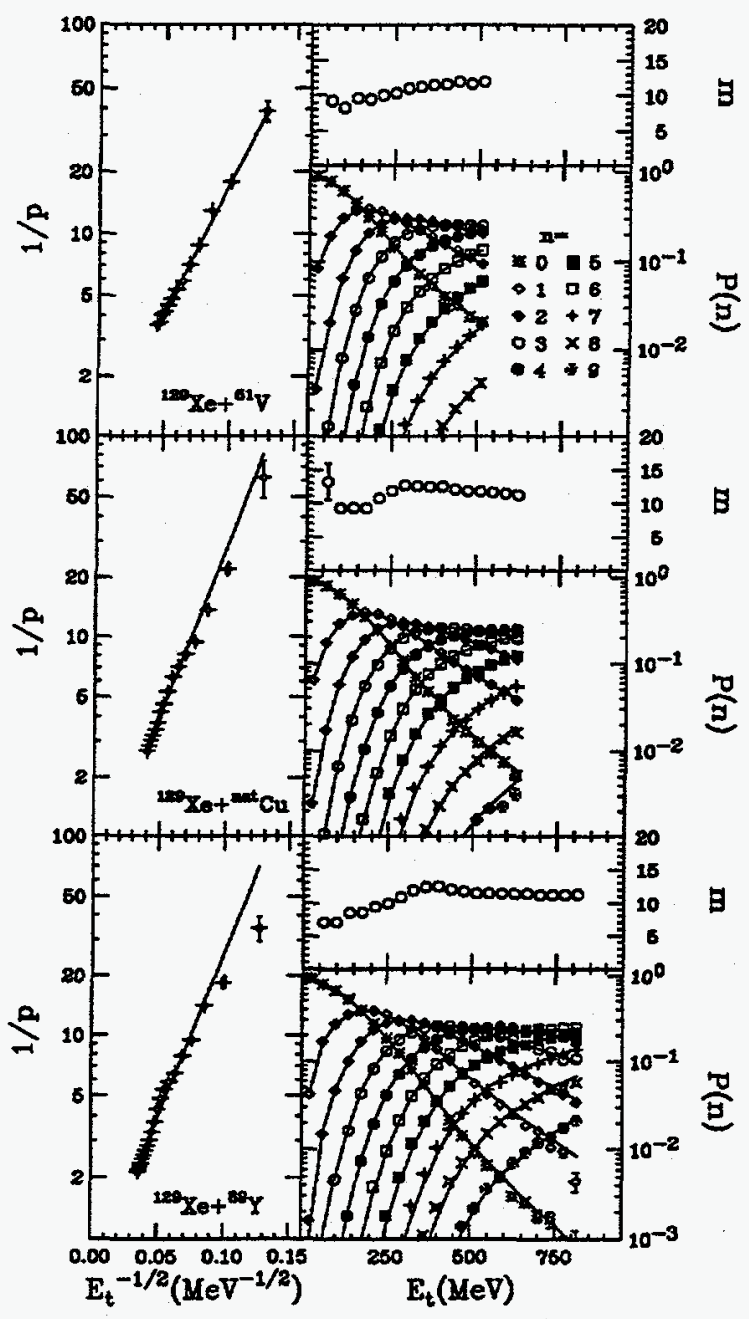

${ }^{36} \mathrm{Ar}+{ }^{187} \mathrm{Au}$

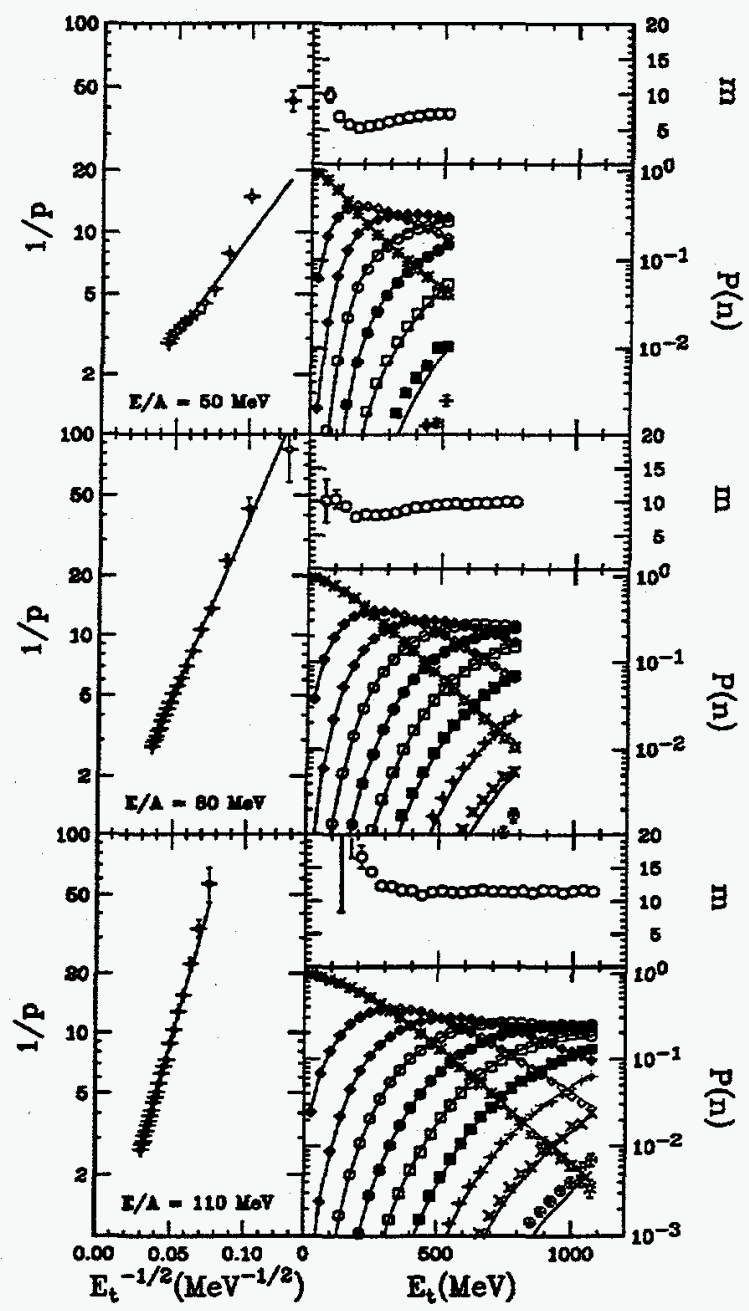

Fig. 1: For the ${ }^{129} \mathrm{Xe}$ induced reactions at $E / A=50 \mathrm{MeV}$ (left figure) and the ${ }^{36} \mathrm{Ar}+{ }^{197} \mathrm{Au}$ reactions at $E / A=50,80$ and $110 \mathrm{MeV}$ (right figure): (left panel) the reciprocal of the single fragment emission probability $1 / p$ as a function of $1 / \sqrt{E_{t}}$; and (right panel) the parameter $m$ (number of the throws in the binomial distribution) and the probability $P(n)$ of emitting $n$ intermediate mass fragments (IMF, $3 \leq Z \leq 20$ ) as a function of the transverse energy $E_{t}$. The solid lines through the excitation functions correspond to binomial distributions calculated with the given values of $m$ and $p$. (See Eq. (1)) 
time separation between fragments. In other words, we can relate the $n$-fragment emission probabilities to the mean time separations between fragments. The validity of this interpretation is testable by experiment.

We have tried to find alternative explanations to the sequential description for the binomial distributions with thermal probabilities. An obvious model is a chain of $m$ links with probability $p$ that any of the links is broken. The probability that $n$ links are broken is given by Eq. (1). This result is, of course, strictly dependent on the dimensionality of the model, and its relevance to multifragmentation is unclear. Nevertheless, it stresses again the fundamental reducibility of the multifragmentation probability to a binary breakup probability $p$.

In summary: 1) The multifragment emission probability has been found to be binomial and reducible to an elementary binary probability. Thus, multifragmentation is empirically reducible to single fragment emission. 2) This binary elementary probability is observed to have a "thermal" energy dependence under the assumption that the excitation energy is proportional to the transversal energy.

\section{Charge Distributions}

These aspects of reducibility and thermal scaling in the integrated fragment emission probabilities lead naturally to the question: Is the charge distribution itself reducible and scalable? In particular, what is the charge distribution form that satisfies the condition of reducibility and of thermal dependence?

Let us first consider the aspect of reducibility as it applies to the charge distributions. In its broadest form, reducibility demands that the probability $p(Z)$, from which an event of $n$ fragments is generated by $m$ trials, is the same at every step of extraction. The consequence of this extreme reducibility is straightforward: the charge distribution for the one-fold events is the same as that for the $n$-fold events and equal to the singles distributions, i.e.:

$$
P_{(1)}(Z)=P_{(n)}(Z)=P_{\text {singles }}(Z)=p(Z) .
$$

We now consider the consequences of the thermal dependence of $p$ [20] on the charge distributions. If the one-fold $=n$-fold $=$ singles distributions is thermal, then

$$
P(Z) \propto e^{-\frac{B(Z)}{T}}
$$

or $T \ln P(Z) \propto-B(Z)$. This suggests that, under the usual assumption $E_{t} \propto E^{*}$ $[20]$, the function

$$
\sqrt{E_{t}} \ln P(Z)=D(Z)
$$

should be independent of $E_{t}$.

In the ${ }^{36} \mathrm{Ar}+{ }^{197} \mathrm{Au}$ reaction, which we now consider here, as in other reactions $[21,22]$, the charge distributions are empirically found to be nearly exponential functions of $Z$ 


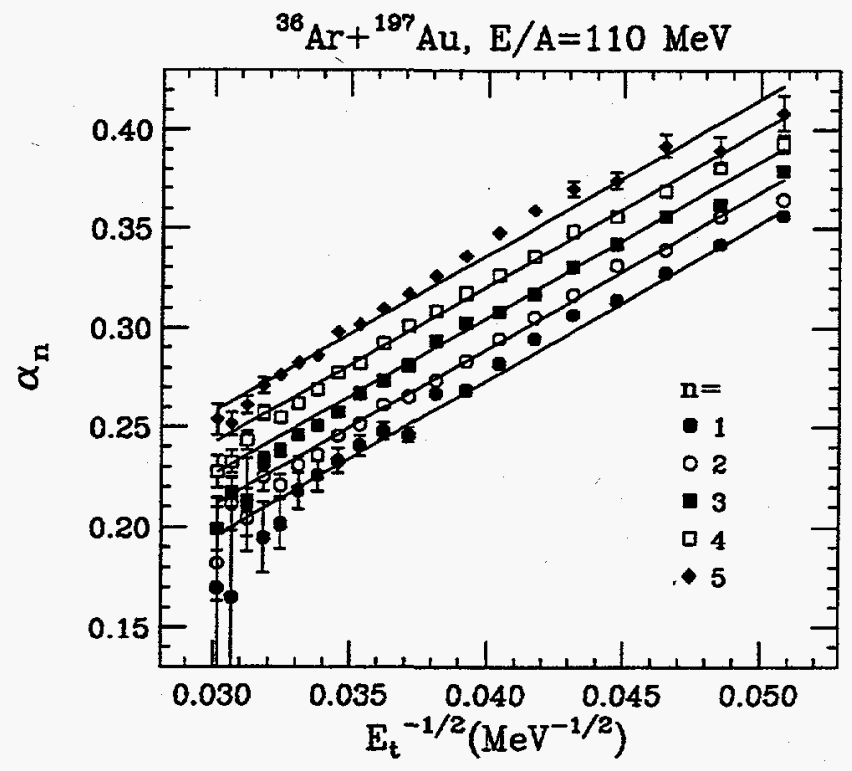

Fig. 2: The exponential fit parameter $\alpha_{n}$ (from fits to the charge distributions, see Eq.(7)) is plotted as a function of $1 / \sqrt{E_{t}}$. The solid lines are a fit to the values of $\alpha_{n}$ using Eq. (9).

$$
P_{n}(Z) \propto e^{-\alpha_{n} Z} .
$$

In light of the above considerations, we would expect for $\alpha_{n}$ the following simple dependence

$$
\alpha_{n} \propto \frac{1}{T} \propto \frac{1}{\sqrt{E_{t}}}
$$

for all folds $n$. Thus a plot of $\alpha_{n}$ vs $1 / \sqrt{E_{t}}$ should give nearly straight lines. This is shown in Fig. 2 for ${ }^{36} \mathrm{Ar}+{ }^{197} \mathrm{Au}$ at $E / A=110 \mathrm{MeV}$.

The expectation of thermal scaling appears to be met quite satisfactorily. For each value of $n$ the exponent $\alpha_{n}$ shows the linear dependence on $1 / \sqrt{E_{t}}$ anticipated in Eq. (8). On the other hand, the extreme reducibility condition demanded by Eq. (4), namely that $\alpha_{1}=\alpha_{2}=\ldots=\alpha_{n}=\alpha$, is not rigorously met. Rather than collapsing on a single straight line, the values of $\alpha_{n}$ for the different fragment multiplicities are offset one with respect to another by what appears to be a small constant quantity.

In fact, one can fit all of the data remarkably well, assuming for $\alpha_{n}$ the form:

$$
\alpha_{n}=\frac{K^{\prime}}{\sqrt{E_{t}}}+n c
$$

which implies:

$$
\alpha_{n}=\frac{K}{T}+n c
$$


or more generally, for the $Z$ distribution:

$$
P_{n}(Z) \propto e^{-\frac{B(Z)}{T}-n c Z} .
$$

Thus, we expect a more general reducibility expression for the charge distribution of any form to be:

$$
\left[\ln P_{n}(Z)+n c Z\right] \sqrt{E_{t}}=F(Z)
$$

for all values of $n$ and $E_{t}$. This equation indicates that it should be possible to reduce the charge distributions associated with any intermediate mass fragment multiplicity to the charge distribution of the singles.

What is the origin of the regular offset that separates the curves in Fig. 2? The general form of Eq. (11) suggests the presence of an entropy term that does not depend explicitly on temperature. The general expression for the free energy (in terms of enthalpy $H$, temperature $T$ and entropy $S$ )

$$
\Delta G=\Delta H(Z)-T \Delta S(Z)
$$

leads to the distribution

$$
P(Z) \propto e^{-\frac{\Delta H(Z)}{T}+\Delta S} .
$$

Typically, $\Delta S$ is of topological or combinatorial origin. For instance, a factor of this sort would appear in the isomerization of a molecule involving a change of symmetry. In our specific case $\Delta S$ may point to an asymptotic combinatorial structure of the multifragmentation process in the high temperature limit. As an example, we consider the Euler problem of an integer to be written as the sum of smaller integers, and calculate the resulting integer distribution. Specifically, let us consider an integer $Z_{0}$ to be broken into $n$ pieces. Let $n_{Z}$ be the number of pieces of size $Z$. The most likely value of $n_{Z}$ can be obtained by extremization of the function [23]

$$
e^{-I}=e^{\sum\left[n_{Z} \ln n_{Z}-n_{Z}\right]+K \sum n_{Z} Z+\gamma \sum n_{Z}}
$$

where the Lagrange multipliers $K$ and $\gamma$ are associated with the constraints

$$
\sum n_{Z} \dot{Z}=Z_{0} ; \sum n_{Z}=n \text {. }
$$

From the extremization we obtain

$$
\frac{\partial I}{\partial n_{Z}}=\ln n_{Z}+K Z+\gamma=0
$$

or

$$
n_{Z}=e^{-K Z-\gamma} .
$$


The constraints now read

$$
\begin{gathered}
Z_{0}=\sum Z e^{-K Z-\gamma} \sim \frac{e^{-\gamma}}{K^{2}} \\
n=\sum e^{-K Z-\gamma} \sim \frac{e^{-\gamma}}{K}
\end{gathered}
$$

from which:

$$
n_{Z}=\frac{n^{2}}{Z_{0}} e^{-\frac{n Z}{Z_{0}}}=c n^{2} e^{-c n Z} .
$$

This expression has the correct asymptotic structure for $T \rightarrow \infty$ required by Eq. (11). The significance of this form is transparent: First, the overall scale for the fragment size is set by the total charge $Z_{0}$. Second, for a specific multiplicity $n$, the scale is reduced by a factor $n$ to the value $Z_{0} / n$.

In summary, we have found for multifragmentation produced in the ${ }^{36} \mathrm{Ar}+{ }^{197} \mathrm{Au}$ reaction at $\mathrm{E} / \mathrm{A}=110 \mathrm{MeV}$ :

1) strong evidence for a thermal scaling of the $Z$-distributions,

2) reducibility of the $n$-fold distributions to the 1-fold distributions by Eq. (11).

3) The structure of the reducibility equation is essentially given by a simple rescaling associated with the multiplicity and the source size.

\section{Phase Coexistence}

While Eq. (21) obviously implies charge conservation, it is not necessary that charge conservation be implemented as suggested by it. In fact it is easy to envisage a regime where the quantity $c$ should be zero. Sequential thermal emission is a case in point. Since any fragment does not know how many other fragments will follow its emission, its charge distribution can not reflect the requirement of an unbiased partition of the total charge among $n$ fragments. We have in mind a liquid drop evaporating fragments of different size and binding energy. "Charge conservation" will affect the distribution minimally, unless evaporation consumes the entire system, and even then, not in the sense of an unbiased partition. A simulation in which fragments with different barriers are allowed to be emitted sequentially according to the binomial scheme of ref. [20] yields indeed $c=0$ if a residue survives.

On the other hand, in a simultaneous emission controlled by a $n$-fragment transition state $[24,25]$, fragments would be strongly aware of each other, and would reflect such an awareness through the charge distribution.

The question then arises whether $c=0$ or $c>0$, or even better, whether one can identify a transition from a regime for which $c=0$ to a new regime for which $c>0$. In order to answer this question, we have studied the charge distributions as a function of fragment multiplicity $n$ and transverse energy $E_{t}$ for a number of systems 


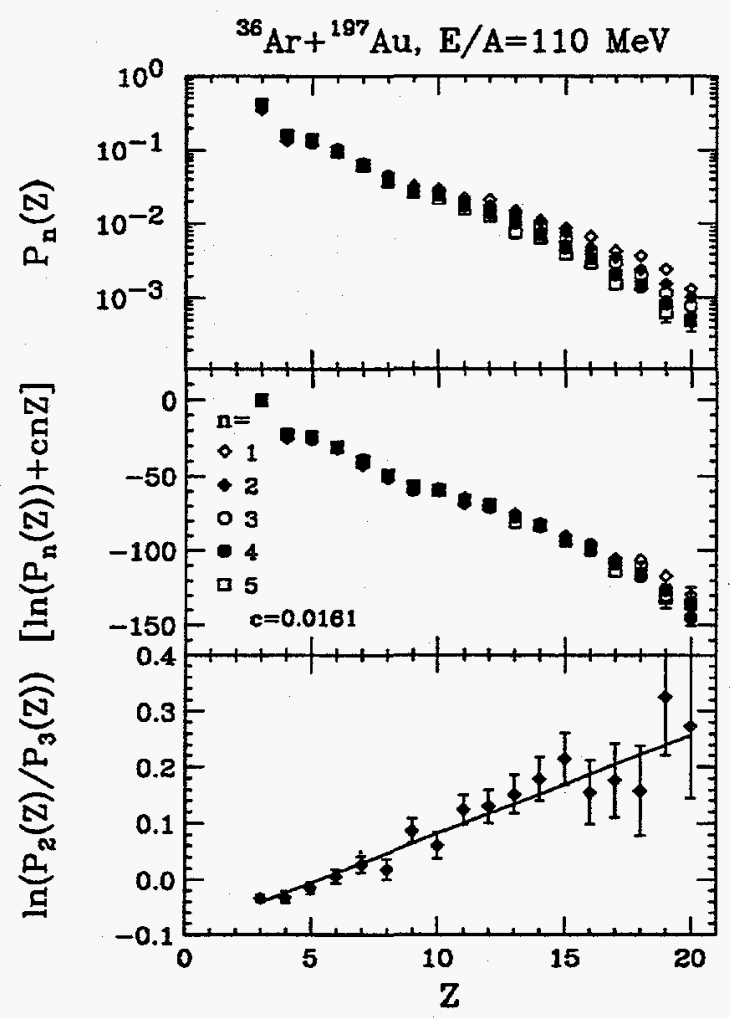

Fig. 3: Top panel: the $n$ gated charge distributions $P_{n}(Z)$ for the reaction ${ }^{36} \mathrm{Ar}+{ }^{197} \mathrm{Au}$ at $E / A=110$ $\mathrm{MeV}$. The charge distributions were constructed from events with $E_{t}=650 \pm 20 \mathrm{MeV}$ and $n=1-5$. Middle panel: the "reduced" charge distribution [26] for the same data using the indicated value of $c$. (The data here are normalized at $Z=3$ ). Bottom panel: the $\log$ of the ratio of $P_{2}(Z) / P_{3}(Z)$. The slope corresponds to $c$ for $n=2$ (see Eq.(22)). The statistical error bars are shown for errors larger than the symbol size. 


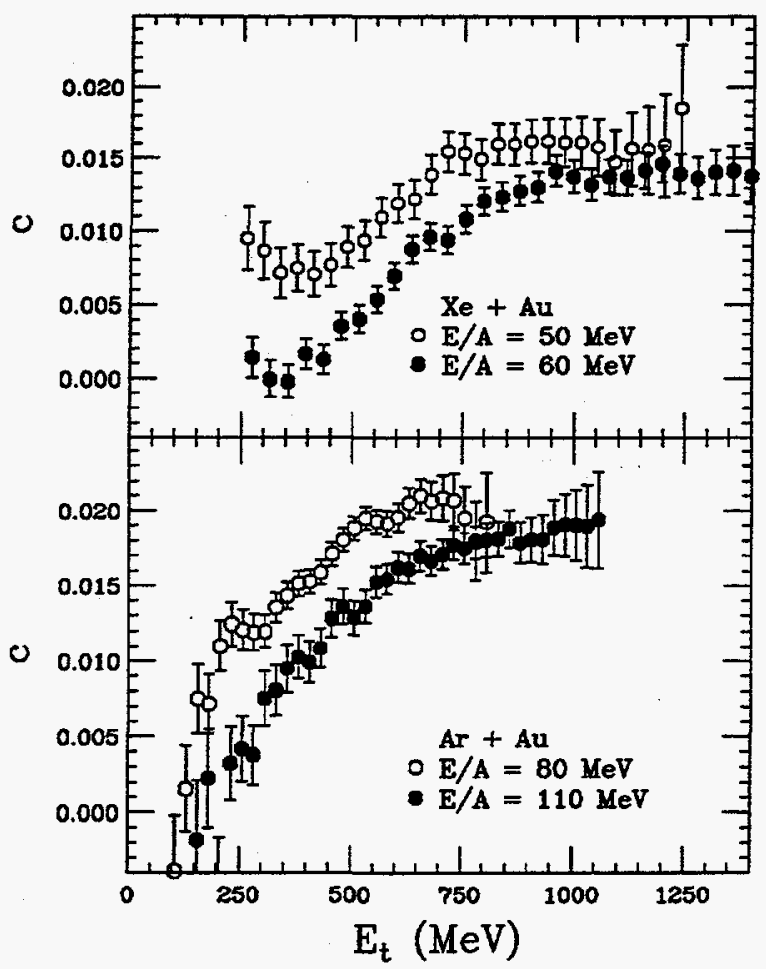

Fig. 4: Plots of the coefficient $c$ versus $E_{t}$ for the reactions ${ }^{129} \mathrm{Xe}+{ }^{197} \mathrm{Au}$ at $E / A=50$ and $60 \mathrm{MeV}$ (top panel) and ${ }^{36} \mathrm{Ar}+{ }^{197} \mathrm{Au}$ at $E / A=80$ and $110 \mathrm{MeV}$ (bottom panel). The error bars are statistical.

and excitation energies. Specifically, we will present data for the reaction ${ }^{36} \mathrm{Ar}+{ }^{197} \mathrm{Au}$ at $E / A=80$ and $110 \mathrm{MeV}$ and the reaction ${ }^{129} \mathrm{Xe}+{ }^{197} \mathrm{Au}$ at $E / A=50$ and $60 \mathrm{MeV}$.

Several approaches were used to extract $c$ from the charge distributions. If the charge distributions are exponential (as is sometimes the case, $P_{n}(Z) \propto e^{-\alpha_{n} Z}$ ), it is sufficient to extract from them the exponential coefficient $\alpha_{n}$. From the $n$ dependence of $\alpha_{n}$, the quantity $c$ is readily extracted [26]. A more general approach which does not depend on any specific form for the charge distribution is to construct at each $E_{t}$ the ratio

$$
\frac{P_{n}(Z)}{P_{n+1}(Z)}=e^{c Z}
$$

A value of $c$ can be extracted for each $n$ by taking the $\log$ of this ratio and finding the slope of the resulting graph (see bottom panel of Fig. 3). A weighted average (over all IMF multiplicities $n$ ) for $c$ can then be constructed at all $E_{t}$. Alternatively, a $\chi^{2}$ can be constructed in terms of the differences in $F(Z)$ (see Eq. (12)) between any pairs of $n$ values and minimized as a function of $c$. These procedures yield essentially the same results. These results are reported in Fig. 4 for the ${ }^{129} \mathrm{Xe}+{ }^{197} \mathrm{Au}$ and ${ }^{36} \mathrm{Ar}+{ }^{197} \mathrm{Au}$ reactions.

It is interesting to notice that for all reactions and bombarding energies the quantity $c$ starts at or near zero, it increases with increasing $E_{t}$ for small $E_{t}$ values, and 


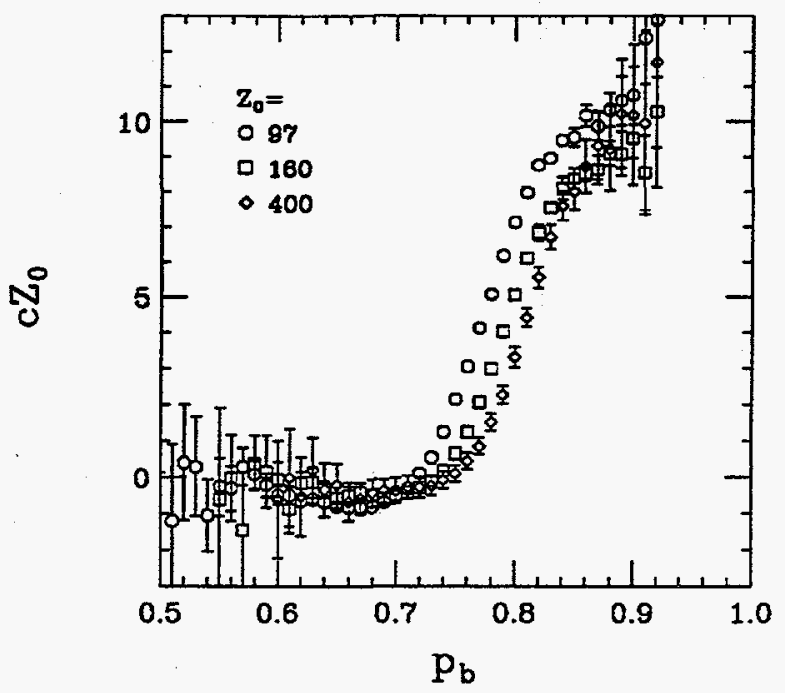

Fig. 5: A plot of $c Z_{0}$ versus the percentage of broken bonds $p_{b}$ from a percolation calculation [27] for three systems $Z_{0}=97$ (circles), $Z_{0}=160$ (squares) and $Z_{0}=400$ (diamonds). The statistical error bars are shown for errors larger than the symbol size.

seems to saturate to a constant value at large $E_{t}$.

This behavior can be compared to that of a fluid crossing from the region of liquid-vapor coexistence to the region of overheated and unsaturated vapor. In the coexistence region, the properties of the saturated vapor cannot depend on the total mass of fluid. The presence of the liquid phase guarantees mass conservation at all average densities for any given temperature. A change in mean density (volume) merely changes the relative amount of the liquid and vapor, without altering the properties of the saturated vapor. Hence the vapor properties, and, in particular, the cluster size distributions cannot reflect the total mass or even the mean density of the system. In our notation, $c=0$.

On the other hand, in the region of unsaturated vapor, there is no liquid to insure mass conservation. Thus the vapor itself must take care of this conservation, at least grand canonically. In our notation, $c>0$.

This description should not be taken too literally, for a variety of reasons, one of which is the finiteness of the system. The $c=0$ regime may signify an evaporative-like emission from a source which survives as a charge conserving residue (liquid), while the $c>0$ regime may signify the complete vaporization of the source.

In order to test these ideas for a finite system, percolation calculations [27] were performed for systems of $Z_{0}=97,160$ and 400 as a function of the percentage of bonds broken $\left(p_{b}\right)$ in the simulation. Values of $c$ were extracted (using Eq.(22)) as a function of $p_{b}$.

The results are shown in Fig. 5. Guided by the insight gleaned from the approximate solution to Euler's problem (see Eq.(21)) we have scaled the extracted values of 
$c$ by the source size $Z_{0}$ in order to remove this leading dependence and to evidentiate the true finite size effects. For values of $p_{b}$ smaller than the critical (percolating) value ( $p_{b}^{\text {crit }} \approx 0.75$ for an infinite system), we find $c=0$. This is the region in which a large (percolating) cluster is present. As $p_{b}$ goes above its critical value, the value of $c$ increases, and eventually saturates in a way very similar to that observed experimentally. Due to the finiteness of the system the transition is smooth rather than sharp and can be made sharper by increasing the size of the system.

Before proceeding, let us remind ourselves that charge conservation is not a finitesize effect. For instance, the chemical potential, introduced in statistical mechanics to conserve mass, survives the thermodynamical limit and retains its meaning for an infinite system, despite the fact that the extensive thermodynamic quantities go to infinity. In our case, while it is true that $c$ goes to zero or that $1 / c$ goes to infinity, it is also true that the product $c Z_{0}$ tends to a finite limit nearly independent of $Z_{0}$.

The significance of the actual experimental value of $c$ in the region where it seems to saturate is unclear. In Eq.(21), $c$ takes a direct meaning for the Euler problem: $c=1 / Z_{0}$. It should be noted that our analysis is not directly comparable to the Euler solution (Eq.(21)) since we have restricted ourselves to a limited region $(3 \leq Z \leq 20)$ of the total charge distribution for our study of how the source is partitioned into different IMF multiplicities. It must also be appreciated that Eq. (21) and the associated dependence of $c$ upon $Z_{0}$ are characteristic of a one-dimensional percolation model. In light of the points mentioned above, it is not unexpected that $c$ appears to be proportional, but not equal, to $1 / Z_{0}$ in the three-dimensional percolation calculation reported in Fig. 5. An interpretation of $c$ in terms of the source size may be possible when more data and a better understanding of the percolation of finite systems are available.

In conclusion:

1) We have presented extensive evidence for an $n$ dependence of charge distributions of the form given by Eq.(12).

2) We have shown that the parameter $c$ increases from near zero at low $E_{t}$ (excitation energy) to a saturation value at high $E_{t}$.

3) Using the analogy of liquid-vapor equilibrium we have argued that $c=0$ may indicate the presence of two phases (liquid-vapor) while $c>0$ may indicate the presence of one phase (unsaturated vapor).

4) We have shown that a percolation calculation carried out for finite systems can be analyzed in the same way as the data, and portrays the same dependence for $c$ as one moves from the region where a percolating cluster is present to one where such a cluster is absent.

\section{Acknowledgements}

This work was supported by the Director, Office of Energy Research, Office of High Energy and Nuclear Physics, Nuclear Physics Division of the US Department of Energy, under contract DE-AC03-76SF00098 and by the National Science Foundation 
under Grant Nos. PHY-8913815, PHY-90117077, and PHY-9214992.

${ }^{a}$ Present address: Instituto Nazionale Fisica Nucleare, V. Amendola, Bari, Italy.

${ }^{b}$ Heavy Ion Laboratory, Warsaw University, PL 02097, Poland

'Present address: Chalk River Laboratories, Chalk River, Ontario K0J 1J0, Canada.

${ }^{d}$ Present address: Instituto de Fisica, Universidade de Sao Paulo, C.P. 20516, CEP 01498, Sao Paulo, Brazil.

${ }^{e}$ GANIL, BP 5027, F-14021 Caen, France

${ }^{f}$ Present address: Indiana University Cyclotron Facility, Bloomington, IN 47405.

${ }^{g}$ Present address: Lawrence Berkeley Laboratory, Berkeley, CA 94720.

${ }^{h}$ Present address: National Laboratory for High Energy Physics, 1-1 Oho, Tsukuba, Ibaraki 305, Japan.

'Present address: Physics Department, Hope College, Holland, MI 49423.

${ }^{j}$ Gesselschaft fur Schwerionenforschung, D-6100 Darmstadt, Germany

${ }^{k}$ Present address: Brookhaven National Laboratory, Upton, NY 19973.

\section{References}

[1] L.G. Sobotka, et. al., Phys. Rev. Lett. 51, 2187 (1983).

[2] L.G. Moretto and G.J. Wozniak, Prog. Part. \& Nucl. Phys. 21, 401 (1988).

[3] D. Guerreau, Formation and Decay of Hot Nuclei: The Experimental Situation ed. (Plenum Publishing Corp., 1989).

[4] D.H.E. Gross, Rep. Prog. Phys. 53, 605 (1990).

[5] L.G. Moretto and G.J. Wozniak, Ann. Rev. Part. Nucl. Sci. 43, 379 (1993).

[6] J. Aichelin, Phys. Rep. 202, 233 (1991).

[7] B. Borderie, Ann. Phys. Fr. 17, 349 (1992).

[8] O. Schapiro and D. H. E. Gross, Nucl. Phys. A 573, 143 (1994).

[9] T. Ethvignot, et al., Phys. Rev. C 48, 618 (1993).

[10] D. Fox, et al., Phys. Rev. C 47, R421 (1993).

[11] E. Bauge, et al., Phys. Rev. Lett. 70, 3705 (1993).

[12] D. R. Bowman, et al., Phys. Rev. Lett. 70, 3534 (1993).

[13] T. C. Sangster, et al., Phys. Rev. C 47, R2457 (1993).

[14] M. Louvel, et. al., Phys. Lett. B 320, 221 (1994).

[15] D. Durand, et al., Phys. Lett. B 345, 397 (1995).

[16] A. Lleres, et al., Phys. Rev. C50, 1973 (1994).

[17] T. Glasmacher, et al., Phys. Rev. C 50, 952 (1994).

[18] L.G. Moretto, D.N. Delis, and G.J. Wozniak, Phys. Rev. Lett. 71, 3935 (1993).

[19] J. Pouliot, et al., Phys. Rev. C 48, 2514 (1993).

[20] L.G. Moretto, et al., Phys. Rev. Lett. 74, 1530 (1995).

[21] Y.D. Kim et al., Phys. Rev. C45, 338 (1992).

[22] D.R. Bowman et al., Phys. Rev. C46, 1834 (1992). 
[23] L.G. Sobotka and L.G. Moretto, Phys. Rev. C31, 668 (1985).

[24] J.A. Lopez and J. Randrup, Nucl. Phys. A 503, 183-222 (1989).

[25] J.A. Lopez and J. Randrup, Nucl. Phys. A 512, 345 (1990).

[26] L. Phair, et al., Phys. Rev. Lett. 75, 213 (1995).

[27] W. Bauer, Phys. Rev. C 38, 1297 (1988). 


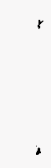

de 